\title{
Double beta decay and the extra-dimensional seesaw mechanism
}

\section{Gautam Bhattacharyya}

Saha Institute of Nuclear Physics, 1/AF Bidhan Nagar, Kolkata 700064, India E-mail: 'gb Qtheory.saha.ernet.in!

\section{Hans Volker Klapdor-Kleingrothaus}

Max-Planck-Institut für Kernphysik, P.O. Box 103980, D-69029 Heidelberg, Germany E-mail: 'klapdor@gustav.mpi-hd.mpg.dé

\section{Heinrich Päs*}

Institut für Theoretische Physik und Astrophysik, Universität Würzburg,

D-97074 Würzburg, Germany

E-mail: paes@physik.uni-wuerzburg.de

\section{Apostolos Pilaftsis}

Department of Physics and Astronomy, University of Manchester,

Manchester M13 9PL, United Kingdom

E-mail: pilaftsi@a35.ph.man.ac.uk

\begin{abstract}
We study the model-building conditions under which an observable $0 \nu \beta \beta$ decay signal is predicted due to Kaluza-Klein singlet neutrinos in theories with large extra dimensions. Our analysis is based on 5-dimensional singlet-neutrino models compactified on an $S^{1} / Z_{2}$ orbifold, where the Standard-Model fields are localized on a 3-brane. We show that sizeable $0 \nu \beta \beta$ rates within the above minimal 5 -dimensional framework would require a non-vanishing shift of the 3 -brane from the orbifold fixed points by an amount smaller than the typical scale $(100 \mathrm{MeV})^{-1}$ characterizing the Fermi nuclear momentum. The resulting 5-dimensional models predict a sizeable effective Majorana-neutrino mass that could be several orders of magnitude larger than the light neutrino masses.
\end{abstract}

\section{Introduction}

Recently, realizations of phenomenologically viable theories with large compact dimensions

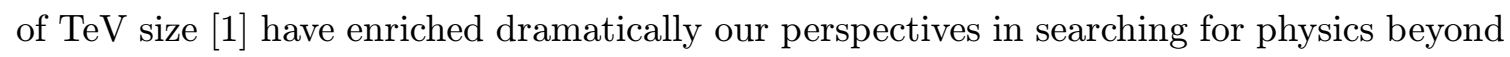

${ }^{*}$ Speaker. 
the Standard Model (SM). Among the possible higher-dimensional realizations, sterile neu-

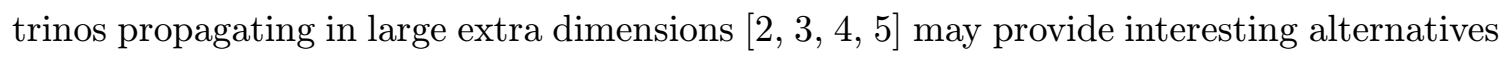
for generating the observed light neutrino masses. On the other hand, detailed experimental studies of neutrino properties may even shed light on the geometry and/or shape of the new dimensions. In this context, one of the most sensitive experimental approaches to neutrino masses and their properties is the search for neutrinoless double beta decay [6메]". Neutrinoless double beta decay, denoted in short as $0 \nu \beta \beta$, corresponds to two single beta decays [i, $]$ occurring simultaneously in one nucleus, thereby converting a nucleus $(Z, A)$ into a nucleus $(Z+2, A)$, i.e.

$$
{ }_{Z}^{A} X \rightarrow{ }_{Z+2}^{A} X+2 e^{-} .
$$

This process violates lepton number by two units and hence its observation would signal physics beyond the SM. To a very good approximation, the half life for a $0 \nu \beta \beta$ decay mediated by light neutrinos is given by

$$
\left[T_{1 / 2}^{0 \nu \beta \beta}\right]^{-1}=\frac{|\langle m\rangle|^{2}}{m_{e}^{2}}\left|\mathcal{M}_{0 \nu \beta \beta}\right|^{2} G_{01}
$$

where $\langle m\rangle$ denotes the effective neutrino Majorana mass, $m_{e}$ is the electron mass and $\mathcal{M}_{0 \nu \beta \beta}$ and $G_{01}$ denote the appropriate nuclear matrix element and the phase space factor,

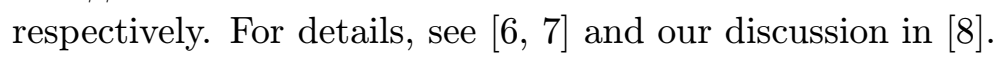

An analysis of the Heidelberg-Moscow experiment reports an evidence for $|\langle m\rangle|=$ $0.39_{-0.34}^{+0.45} \mathrm{eV} \quad(95 \% \mathrm{CL})[\overline{9}]$. The IGEX experiment derives a bound of $|\langle m\rangle|<0.33-$ $1.35 \mathrm{eV}$ (95\% CL) [1] $\left.{ }_{1}^{1}\right]$. The NEMO3 experiment, which may come to a sensitivity in this range, has started operation [1] 1] $1_{1}^{1}$. Several proposals for next-generation experiments aim at sensitivities down to (few) $\times 10^{-2} \mathrm{eV}$, among these the GENIUS, CUORE and EXO projects with approved prototypes (see [1] $\left.{ }_{1}^{1} \overline{2}\right]$ for an overview).

Here, we focus on the question, whether extra dimensional neutrino models can be tested by double beta experiments via an observable signal in present or next generation setups. Within the framework of theories with large extra dimensions, previous studies on neutrinoless double beta decays were performed within the context of higher-dimensional models that utilize the shining mechanism from a distant brane [1] $\overline{3}$ i] and of theories with

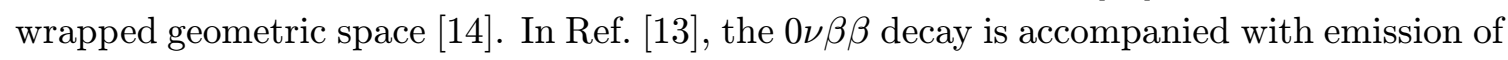
Majorons, whereas the prediction in [1] $\left.{ }_{1}^{1}\right]$ falls short by two orders of magnitude to account for an observable signal in running experiments.

Here we consider an even more minimal higher-dimensional framework of leptonnumber violation, namely 5 -dimensional theories compactified on a $S^{1} / Z_{2}$ orbifold, in which only one 5-dimensional (bulk) sterile neutrino is added to the field content of the SM [8] In this minimal model, the SM fields are localized on a 4-dimensional Minkowski subspace, also termed 3-brane. The model naturally generates small neutrino masses in an higher-dimensional analogue of the seesaw mechanism [i2.

\section{Minimal higher-dimensional neutrino models}

In this section, we will describe the basic low-energy structure of minimal higher-dimensional 


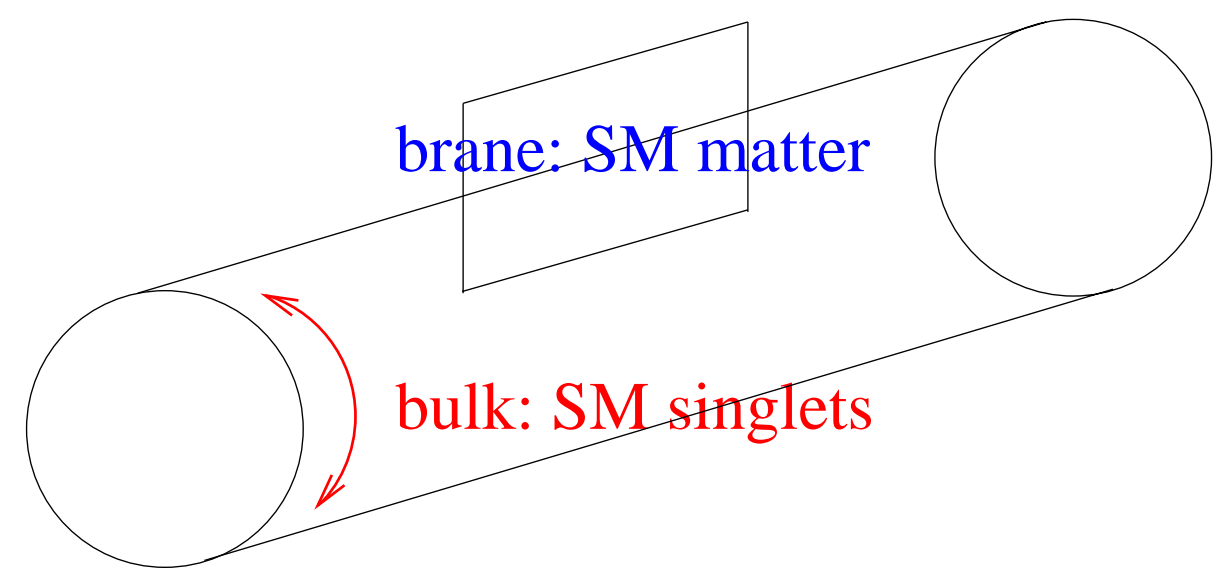

Figure 1: The SM matter is localized on a 3-brane, while the sterile singlet neutrinos are allowed to propagate in the bulk. This frameowork naturally generates small neutrino masses.

extensions of the SM that include singlet neutrinos. In particular, we assume that singlet neutrinos being neutral under the $\mathrm{SU}(2)_{L} \otimes \mathrm{U}(1)_{Y}$ gauge group can freely propagate in a higher-dimensional space of $[1+(3+\delta)]$ dimensions, the so-called bulk, whereas all SM particles are localized in a $(1+3)$-dimensional subspace, known as 3-brane or simply brane. However, even singlet neutrinos themselves may live in a subspace of an even higherdimensional space of $\left[1+\left(3+n_{g}\right)\right]$ dimensions, with $\delta \leq n_{g}$, in which gravity propagates.

We shall restrict our study to 5 -dimensional models, i.e. the case $\delta=1$, where the singlet neutrinos are compactified on a $S^{1} / Z_{2}$ orbifold. Specifically, the leptonic sector of our 5-dimensional model consists of the SM lepton fields:

$$
L(x)=\left(\begin{array}{c}
\nu_{l}(x) \\
l_{L}(x)
\end{array}\right), \quad l_{R}(x),
$$

with $l=e, \mu, \tau$, and one 5-dimensional (bulk) singlet neutrino:

$$
N(x, y)=\left(\begin{array}{l}
\xi(x, y) \\
\bar{\eta}(x, y)
\end{array}\right),
$$

where $y$ denotes the additional compact dimension, and $\xi$ and $\eta$ are 5-dimensional twocomponent spinors. For generality, we will assume that the brane, where the SM leptons are localized, is shifted from the orbifold fixed point $y=0$ to $y=a$.

As usual, we impose the periodic boundary condition $N(x, y)=N(x, y+2 \pi R)$ with respect to $y$ dimension on the singlet neutrino field. In addition, the action of $S^{1} / Z_{2}$ orbifolding on the 5-dimensional spinors $\xi$ and $\eta$ entails the additional identifications:

$$
\xi(x, y)=\xi(x,-y), \quad \eta(x, y)=-\eta(x,-y)
$$

In other words, the spinors $\xi$ and $\eta$ are symmetric and antisymmetric under a $y$ reflection, respectively. 
With the above definitions, the most generic effective 4-dimensional Lagrangian of such a model is given by $[2$, int.

$$
\begin{aligned}
\mathcal{L}_{\text {eff }}= & \int_{0}^{2 \pi R} d y\left\{\bar{N}\left(i \gamma^{\mu} \partial_{\mu}+\gamma_{5} \partial_{y}\right) N-\frac{1}{2}\left(M N^{T} C^{(5)-1} N+\text { h.c. }\right)\right. \\
& \left.+\delta(y-a)\left[\frac{h_{1}^{l}}{\left(M_{F}\right)^{\delta / 2}} L \tilde{\Phi}^{*} \xi+\frac{h_{2}^{l}}{\left(M_{F}\right)^{\delta / 2}} L \tilde{\Phi}^{*} \eta+\text { h.c. }\right]+\delta(y-a) \mathcal{L}_{\mathrm{SM}}\right\},
\end{aligned}
$$

where $\tilde{\Phi}=i \sigma_{2} \Phi^{*}$ is the hypercharge-conjugate of the SM Higgs doublet $\Phi$, with hypercharge $Y(\Phi)=1$, and $\mathcal{L}_{\mathrm{SM}}$ denotes the SM Lagrangian which is restricted on a brane at $y=a$ [2] In addition, $M_{F}$ is the fundamental $n_{g}$-dimensional Planck scale and $\delta=1$ for sterile neutrinos propagating in 5 dimensions. Notice that the mass term $m_{D} \bar{N} N$ is not allowed in (1) $\left(\begin{array}{l}2 \\ 2\end{array}\right)$, as a result of the $Z_{2}$ discrete symmetry.

We now proceed with the compactification of the $y$ dimension of the $S^{1} / Z_{2}$ orbifold model. Because of their symmetric and antisymmetric properties $(\overline{2} . \overline{3})$ under $y$ reflection, the two-component spinors $\xi$ and $\eta$ can be expanded in a Fourier series of cosine and sine harmonics:

$$
\begin{aligned}
& \xi(x, y)=\frac{1}{\sqrt{2 \pi R}} \xi_{0}(x)+\frac{1}{\sqrt{\pi R}} \sum_{n=1}^{\infty} \xi_{n}(x) \cos \left(\frac{n y}{R}\right) \\
& \eta(x, y)=\frac{1}{\sqrt{\pi R}} \sum_{n=1}^{\infty} \eta_{n}(x) \sin \left(\frac{n y}{R}\right)
\end{aligned}
$$

where the chiral spinors $\xi_{n}(x)$ and $\eta_{n}(x)$ form an infinite tower of KK modes.

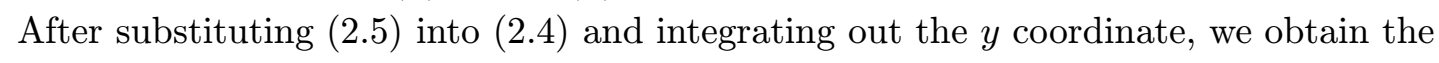
effective 4-dimensional Lagrangian

$$
\begin{aligned}
\mathcal{L}_{\text {eff }}= & \mathcal{L}_{\mathrm{SM}}+\bar{\xi}_{0}\left(i \bar{\sigma}^{\mu} \partial_{\mu}\right) \xi_{0}+\left(\bar{h}_{1}^{l(0)} L \tilde{\Phi}^{*} \xi_{0}-\frac{1}{2} M \xi_{0} \xi_{0}+\text { h.c. }\right)+\sum_{n=1}^{\infty}\left[\bar{\xi}_{n}\left(i \bar{\sigma}^{\mu} \partial_{\mu}\right) \xi_{n}\right. \\
& +\bar{\eta}_{n}\left(i \bar{\sigma}^{\mu} \partial_{\mu}\right) \eta_{n}+\frac{n}{R}\left(\xi_{n} \eta_{n}+\bar{\xi}_{n} \bar{\eta}_{n}\right)-\frac{1}{2} M\left(\xi_{n} \xi_{n}+\bar{\eta}_{n} \bar{\eta}_{n}+\text { h.c. }\right) \\
& \left.+\sqrt{2}\left(\bar{h}_{1}^{l(n)} L \tilde{\Phi}^{*} \xi_{n}+\bar{h}_{2}^{l(n)} L \tilde{\Phi}^{*} \eta_{n}+\text { h.c. }\right)\right]
\end{aligned}
$$

where

$$
\begin{aligned}
& \bar{h}_{1}^{l(n)}=\frac{h_{1}^{l}}{\left(2 \pi M_{F} R\right)^{\delta / 2}} \cos \left(\frac{n a}{R}\right)=\left(\frac{M_{F}}{M_{\mathrm{P}}}\right)^{\delta / n_{g}} h_{1}^{l} \cos \left(\frac{n a}{R}\right), \\
& \bar{h}_{2}^{l(n)}=\frac{h_{2}^{l}}{\left(2 \pi M_{F} R\right)^{\delta / 2}} \sin \left(\frac{n a}{R}\right)=\left(\frac{M_{F}}{M_{\mathrm{P}}}\right)^{\delta / n_{g}} h_{2}^{l} \sin \left(\frac{n a}{R}\right) .
\end{aligned}
$$

In deriving the last step on the RHS's of $\left(\overline{2}_{2} . \overline{8}_{i}^{\prime}\right)$ and $\left(\overline{2}_{2}^{2} \cdot \overline{9}_{i}^{i}\right)$, we have employed the basic relation among the Planck mass $M_{\mathrm{P}}$, the corresponding $n_{g}$-dimensional Planck mass $M_{F}$ and the compactification radii $R$ (all taken to be of equal size):

$$
M_{\mathrm{P}}=\left(2 \pi M_{F} R\right)^{n_{g} / 2} M_{F} .
$$


From $\left(\overline{2} . \overline{8}_{1}^{\prime}\right)$ and $\left(\overline{2}_{2}^{2} . \overline{9}\right)$, we see that the reduced 4-dimensional Yukawa couplings $\bar{h}_{1,2}^{(n)}$ can be suppressed by many orders of magnitude [3, in the quantum gravity scale $M_{F}$. Thus, if gravity and bulk neutrinos feel the same number of extra dimensions, i.e. $\delta=n_{g}$, the 4-dimensional Yukawa couplings $\bar{h}_{1}^{(n)}$ and $\bar{h}_{2}^{(n)}$ are naturally suppressed by a huge factor $M_{F} / M_{\mathrm{P}} \sim 10^{-15}$, for $M_{F} \approx 10 \mathrm{TeV}$. From $\left(\overline{2}_{2} \cdot \overline{2}_{i}^{2}\right)$, we observe that $\xi$ and $\bar{\eta}$ belong to the same multiplet and hence have the same lepton number. It then follows from $\left(2 \bar{i}_{1}\right)$ that the simultaneous presence of $\bar{h}_{1}^{(n)}$ and $\bar{h}_{2}^{(n)}$ in an amplitude gives rise to lepton number violation by two units.

If the brane were located at the one of the two orbifold fixed points, e.g. at $y=0$, the operator $L \tilde{\Phi}^{*} \eta$ would be absent as a consequence of the $Z_{2}$ discrete symmetry. However, if the brane is shifted by an amount $a \neq 0$, the above operator is no longer absent. In fact, the coexistence of the two operators $L \tilde{\Phi}^{*} \xi$ and $L \tilde{\Phi}^{*} \eta$ breaks the lepton number leading to observable effects in neutrinoless double beta decay experiments.

Let us now introduce the weak basis for the KK-Weyl spinors

$$
\chi_{ \pm n}=\frac{1}{\sqrt{2}}\left(\xi_{n} \pm \eta_{n}\right) .
$$

Following [i2] smallest diagonal entry of the KK neutrino mass matrix is $|\varepsilon|=\min \left(\left|M-\frac{k}{R}\right|\right) \leq 1 /(2 R)$, for a given value $k=k_{0}$. In this newly defined basis, the effective kinetic Lagrangian becomes

$$
\mathcal{L}_{\text {kin }}=\frac{1}{2} \bar{\Psi}_{\nu}\left(i \not \partial-\mathcal{M}_{\nu}^{\mathrm{KK}}\right) \Psi_{\nu}
$$

where $\Psi_{\nu}$ is the reordered (4-component) Majorana-spinor vector

$$
\Psi_{\nu}^{T}=\left[\left(\begin{array}{c}
\nu_{l} \\
\bar{\nu}_{l}
\end{array}\right),\left(\begin{array}{c}
\chi_{k_{0}} \\
\bar{\chi}_{k_{0}}
\end{array}\right),\left(\begin{array}{c}
\chi_{k_{0}+1} \\
\bar{\chi}_{k_{0}+1}
\end{array}\right),\left(\begin{array}{c}
\chi_{k_{0}-1} \\
\bar{\chi}_{k_{0}-1}
\end{array}\right), \cdots,\left(\begin{array}{c}
\chi_{k_{0}+n} \\
\bar{\chi}_{k_{0}+n}
\end{array}\right),\left(\begin{array}{c}
\chi_{k_{0}-n} \\
\bar{\chi}_{k_{0}-n}
\end{array}\right), \cdots\right]
$$

and $\mathcal{M}_{\nu}^{\mathrm{KK}}$ the corresponding $\mathrm{KK}$ neutrino mass matrix

$$
\mathcal{M}_{\nu}^{\mathrm{KK}}=\left(\begin{array}{ccccccc}
0 & m & m & m & m & m & \cdots \\
m & \varepsilon & 0 & 0 & 0 & 0 & \cdots \\
m & 0 & \varepsilon+\frac{1}{R} & 0 & 0 & 0 & \cdots \\
m & 0 & 0 & \varepsilon-\frac{1}{R} & 0 & 0 & \cdots \\
m & 0 & 0 & 0 & \varepsilon+\frac{2}{R} & 0 & \cdots \\
m & 0 & 0 & 0 & 0 & \varepsilon-\frac{2}{R} & \cdots \\
\vdots & \vdots & \vdots & \vdots & \vdots & \vdots & \ddots
\end{array}\right)
$$

with $m=v \bar{h}_{1} / \sqrt{2}$. In a three-generation model, $m$ and $\bar{h}_{1}$ are both 3 -vectors in the flavour space, i.e. $\bar{h}_{1}=\left(\bar{h}_{1}^{e}, \bar{h}_{1}^{\mu}, \bar{h}_{1}^{\tau}\right)^{T}$. For simplicity we assume here that $\bar{h}_{1}=\bar{h}_{1}^{e}$.

The eigenvalues of $\mathcal{M}_{\nu}^{\mathrm{KK}}$ can be computed from the characteristic eigenvalue equation $\operatorname{det}\left(\mathcal{M}_{\nu}^{\mathrm{KK}}-\lambda \mathbf{1}\right)=0$, which is analytically given by

$$
\prod_{n=0}^{\infty}\left[(\lambda-\varepsilon)^{2}-\frac{n^{2}}{R^{2}}\right]\left[1+\frac{\varepsilon}{\lambda-\varepsilon}-m^{2} \sum_{n=-\infty}^{\infty} \frac{1}{(\lambda-\varepsilon)^{2}-\frac{n^{2}}{R^{2}}}\right]=0 .
$$


Since it can be shown that $\lambda-\varepsilon= \pm n / R$ is never an exact solution to the characteristic equation, only the second factor in (2.15i) can vanish. Employing complex contour integration techniques, the summation in the second factor in (2.15) can be performed exactly, leading to an equivalent transcendental equation

$$
\lambda=\pi m^{2} R \cot [\pi R(\lambda-\varepsilon)] .
$$

As was already discussed in [2], if $\varepsilon=0$, (20) implies that the mass spectrum consists of massive KK Majorana neutrinos degenerate in pairs with opposite CP parities. If $\varepsilon=$ $1 /(2 R)$, the KK mass spectrum contains a massless state, which is predominantly lefthanded if $m R<1$, while the remaining massive KK states form degenerate pairs with opposite CP parities, exactly as in the $\varepsilon=0$ case. However, if $\varepsilon \neq 0,1 /(2 R)$, the lepton number gets broken. ${ }^{1}$ In this case, there is no massless state in the spectrum, and the above exact degeneracy among the massive Majorana neutrinos becomes only approximate, with a mass splitting of order $2 \varepsilon$ for each would-be $(\varepsilon \rightarrow 0)$ degenerate KK pair.

We now consider an orbifold model, in which the $y=0$ brane is displaced from the orbifold fixed points by an amount $a$. Under certain restrictions in Type I string theory [i] i2i], such an operation can be performed respecting the $Z_{2}$ invariance of the original higherdimensional action. In particular, one can take explicitly account of this last property by considering the following replacements in the effective Lagrangian (2.24):

$$
\begin{aligned}
\xi \delta(y-a) & \rightarrow \frac{1}{2} \xi[\delta(y-a)+\delta(y+a-2 \pi R)], \\
\eta \delta(y-a) & \rightarrow \frac{1}{2} \eta[\delta(y-a)-\delta(y+a-2 \pi R)],
\end{aligned}
$$

with $0 \leq a<\pi R$ and $0 \leq y \leq 2 \pi R$. It is obvious that a $Z_{2}$-invariant implementation of brane-shifted couplings requires the existence of two branes at least, placed at $y=a$ and $y=2 \pi R-a$.

Proceeding as above, the effective KK neutrino mass matrix $\mathcal{M}_{\nu}^{\mathrm{KK}}$ for the orbifold model with a shifted brane can be written down in an analogous form

$$
\mathcal{M}_{\nu}^{\mathrm{KK}}=\left(\begin{array}{ccccccc}
0 & m^{(0)} & m^{(1)} & m^{(-1)} & m^{(2)} & m^{(-2)} & \ldots \\
m^{(0)} & \varepsilon & 0 & 0 & 0 & 0 & \ldots \\
m^{(1)} & 0 & \varepsilon+\frac{1}{R} & 0 & 0 & 0 & \ldots \\
m^{(-1)} & 0 & 0 & \varepsilon-\frac{1}{R} & 0 & 0 & \ldots \\
m^{(2)} & 0 & 0 & 0 & \varepsilon+\frac{2}{R} & 0 & \ldots \\
m^{(-2)} & 0 & 0 & 0 & 0 & \varepsilon-\frac{2}{R} & \ldots \\
\vdots & \vdots & \vdots & \vdots & \vdots & \vdots & \ddots
\end{array}\right),
$$

where

$$
m^{(n)}=\frac{v}{\sqrt{2}}\left[\bar{h}_{1} \cos \left(\frac{\left(n-k_{0}\right) a}{R}\right)+\bar{h}_{2} \sin \left(\frac{\left(n-k_{0}\right) a}{R}\right)\right]=m \cos \left(\frac{n a}{R}-\phi_{h}\right),
$$

\footnotetext{
${ }^{1}$ Alternatively, lepton number may also be broken through the Scherk-Schwarz mechanism [i] the Scherk-Schwarz rotation angle will induce terms very similar to those depending on $\varepsilon$ [2]
} 
with $m=v \sqrt{\left(\bar{h}_{1}^{2}+\bar{h}_{2}^{2}\right) / 2}$ and $\phi_{h}=\tan ^{-1}\left(\bar{h}_{2} / \bar{h}_{1}\right)+k_{0} a / R$. As before, we consider an one-generation model with $\bar{h}_{1}=\bar{h}_{1}^{e}$ and $\bar{h}_{2}=\bar{h}_{2}^{e}$, which renders the analytic determination of the eigenvalue equation tractable. Thus, for our one-generation brane-shifted model, the characteristic eigenvalue equation reads

$$
\prod_{n=0}^{\infty}\left[(\lambda-\varepsilon)^{2}-\frac{n^{2}}{R^{2}}\right]\left[1+\frac{\varepsilon}{\lambda-\varepsilon}-\frac{1}{\lambda-\varepsilon} \sum_{n=-\infty}^{\infty} \frac{m^{(n) 2}}{\lambda-\varepsilon-\frac{n}{R}}\right]=0,
$$

which is equivalent to

$$
\lambda=\sum_{n=-\infty}^{\infty} \frac{m^{(n) 2}}{\lambda-\varepsilon-\frac{n}{R}} .
$$

We carry out the infinite sum in $(\overline{2} . \overline{1})$ analytically and derive the eigenvalue equation for the simplest class of cases, where $a=\pi R / q$ with $q$ an integer larger than 1 , i.e. $q \geq 2$. More precisely, we find

$$
\lambda=\pi m^{2} R\left\{\cos ^{2}\left[\phi_{h}-a(\lambda-\varepsilon)\right] \cot [\pi R(\lambda-\varepsilon)]-\frac{1}{2} \sin \left[2 \phi_{h}-2 a(\lambda-\varepsilon)\right]\right\}
$$

Observe that unless $\varepsilon=1 /(2 R), a=\pi R / 2$ and $\phi_{h}=\pi / 4$, the mass spectrum consists of massive non-degenerate KK neutrinos. However, it can be shown from (2.22) that this tree-level mass splitting between a pair of KK Majorana neutrinos is generally small for $m_{(n)} \gg 1 / R$. In particular, this tree-level mass splitting is almost independent of $a$ and subleading so as to play any relevant rôle in our calculations.

\section{Effective neutrino-mass estimates}

In this section, we calculate the $0 \nu \beta \beta$ observable $\langle m\rangle$ in orbifold 5 -dimensional models. This quantity determines the size of the neutrinoless double beta decay amplitude, which is induced by $W$-boson exchange graphs. To this end, it is important to know the interactions of the $W^{ \pm}$bosons to the charged leptons $l=e, \mu, \tau$ and the KK-neutrino mass-eigenstates

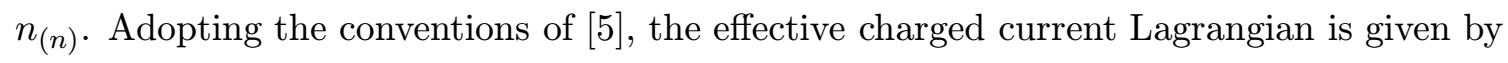

$$
\mathcal{L}_{\text {int }}^{W^{ \pm}}=-\frac{g_{w}}{\sqrt{2}} W^{-\mu} \sum_{l=e, \mu, \tau}\left(B_{l \nu_{l}} \bar{l} \gamma_{\mu} P_{L} \nu_{l}+\sum_{n=-\infty}^{+\infty} B_{l, n} \bar{l} \gamma_{\mu} P_{L} n_{(n)}\right)+\text { h.c. }
$$

where $g_{w}$ is the weak coupling constant, $P_{L}=\left(1-\gamma_{5}\right) / 2$ is the left-handed chirality projector, and $B$ is an infinite dimensional mixing matrix. The matrix $B$ satisfies the following crucial identities:

$$
\begin{aligned}
B_{l \nu_{l}} B_{l^{\prime} \nu_{l}}^{*}+\sum_{n=-\infty}^{+\infty} B_{l, n} B_{l^{\prime}, n}^{*} & =\delta_{l l^{\prime}}, \\
B_{l \nu_{l}} m_{\nu_{l}} B_{l^{\prime} \nu_{l}}+\sum_{n=-\infty}^{+\infty} B_{l, n} m_{(n)} B_{l^{\prime}, n} & =0 .
\end{aligned}
$$


Equation (13) reflects the unitarity properties of the charged lepton weak space, and (13.3) holds true, as a result of the absence of the Majorana mass terms $\nu_{l} \nu_{l^{\prime}}$ from the effective Lagrangian in the flavour basis. For the models under discussion, the KK neutrino masses $m_{(n)}$ can be determined exactly by the solutions of the corresponding transcendental equations. To a good approximation, however, these solutions for large $n$ simplify to

$$
m_{(n)} \approx \frac{n}{R}+\varepsilon
$$

Like the neutrino masses, the mixing-matrix elements $B_{e \nu}$ and $B_{e, n}$ can also be computed exactly [īin]:

$$
\begin{aligned}
& B_{e \nu}=\frac{1}{1+\pi^{2} m^{2} R^{2}+\frac{m_{\nu}^{2}}{m^{2}}}, \\
& B_{e, n} \simeq \frac{m^{2} \cos ^{2}\left(\frac{n a}{R}-\phi_{h}\right)}{\left(\frac{n}{R}+\varepsilon\right)^{2}} .
\end{aligned}
$$

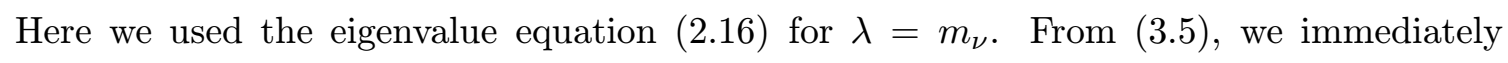
see that if $m R \ll 1$ and $m_{\nu} \ll m$, it is $B_{e \nu} \approx 1$ and hence the lightest neutrino state is predominantly left-handed. The last approximate equality in ( $\left(\overline{3}_{-} . \overline{6}_{i}\right)$ corresponds to a large $n$.

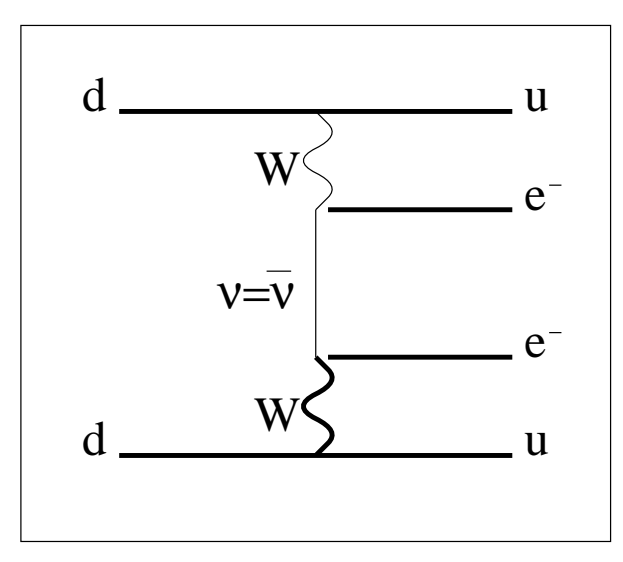

Figure 2: Feynman diagram for neutrinoless double beta decay. In the extradimensional framework, all KK states contribute to the propagator, weighted with the appropriate mixing and nuclear matrix elements.

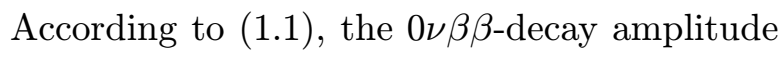
$\mathcal{T}_{0 \nu \beta \beta}$ is given by [i]

$$
\mathcal{T}_{0 \nu \beta \beta}=\frac{\langle m\rangle}{m_{e}} \mathcal{M}_{\mathrm{GTF}}\left(m_{\nu}\right)
$$

where $\mathcal{M}_{\mathrm{GTF}}=\mathcal{M}_{\mathrm{GT}}-\mathcal{M}_{\mathrm{F}}$ is the difference of the nuclear matrix elements for the so-called GamowTeller and Fermi transitions. Note that this difference of nuclear matrix elements sensitively depends on the mass of the exchanged KK neutrino in a $0 \nu \beta \beta$ decay (compare the discussion in pecially if the exchanged KK-neutrino mass $m_{(n)}$ is comparable or larger than the characteristic Fermi nuclear momentum $q_{F} \approx 100 \mathrm{MeV}$, the nuclear matrix element $\mathcal{M}_{\mathrm{GTF}}$ decreases as $1 / m_{(n)}^{2}$. The general expression for the effective Majorana-neutrino mass $\langle m\rangle$ in $(\overline{2} \overline{2} . \overline{7} \bar{i})$ is given by

$$
\langle m\rangle=\frac{1}{\mathcal{M}_{\mathrm{GTF}}\left(m_{\nu}\right)} \sum_{n=-\infty}^{\infty} B_{e, n}^{2} m_{(n)}\left[\mathcal{M}_{\mathrm{GTF}}\left(m_{(n)}\right)-\mathcal{M}_{\mathrm{GTF}}\left(m_{\nu}\right)\right] .
$$

In the above, the first term describes the genuine higher-dimensional effect of KK-neutrino exchanges, while the second term is the standard contribution of the light neutrino $\nu$, rewritten by virtue of (13.3). Note that the dependence of the nuclear matrix element

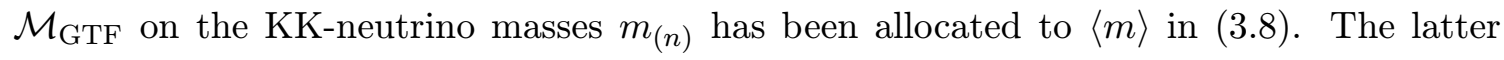




\begin{tabular}{|c|c|c|c|c|}
\hline \multirow{2}{*}{$m_{(n)}[\mathrm{MeV}]$} & \multicolumn{4}{|c|}{$\mathcal{M}_{\mathrm{GTF}}\left(m_{(n)}\right)$} \\
\hline & $76 \mathrm{Ge}$ & ${ }^{82} \mathrm{Se}$ & ${ }^{100} \mathrm{Mo}$ & ${ }^{116} \mathrm{Cd}$ \\
\hline$\leq 1$ & 4.33 & 4.03 & 4.86 & 3.29 \\
10 & 4.34 & 4.04 & 4.81 & 3.29 \\
$10^{2}$ & 3.08 & 2.82 & 3.31 & 2.18 \\
$10^{3}$ & $1.40 \times 10^{-1}$ & $1.25 \times 10^{-1}$ & $1.60 \times 10^{-1}$ & $9.34 \times 10^{-2}$ \\
$10^{4}$ & $1.39 \times 10^{-3}$ & $1.24 \times 10^{-3}$ & $1.60 \times 10^{-3}$ & $9.26 \times 10^{-4}$ \\
$10^{5}$ & $1.39 \times 10^{-5}$ & $1.24 \times 10^{-5}$ & $1.60 \times 10^{-5}$ & $9.26 \times 10^{-6}$ \\
$10^{6}$ & $1.39 \times 10^{-7}$ & $1.24 \times 10^{-7}$ & $1.60 \times 10^{-7}$ & $9.26 \times 10^{-8}$ \\
$10^{7}$ & $1.39 \times 10^{-9}$ & $1.24 \times 10^{-9}$ & $1.60 \times 10^{-9}$ & $9.26 \times 10^{-10}$ \\
\hline \hline & \multicolumn{4}{|c|}{} \\
$m_{(n)}[\mathrm{MeV}]$ & \multicolumn{4}{|c|}{$\mathcal{M}_{\mathrm{GTF}}\left(m_{(n)}\right)$} \\
\hline & $128 \mathrm{Te}$ & $130 \mathrm{Te}$ & $136 \mathrm{Xe}$ & $150 \mathrm{Nd}$ \\
\hline$\leq 1$ & 4.50 & 3.89 & 1.83 & 5.30 \\
10 & 4.52 & 3.91 & 1.88 & 5.45 \\
$10^{2}$ & 3.19 & 2.79 & 1.48 & 4.24 \\
$10^{3}$ & $1.46 \times 10^{-1}$ & $1.29 \times 10^{-1}$ & $7.07 \times 10^{-2}$ & $2.02 \times 10^{-1}$ \\
$10^{4}$ & $1.46 \times 10^{-3}$ & $1.28 \times 10^{-3}$ & $7.04 \times 10^{-4}$ & $2.02 \times 10^{-3}$ \\
$10^{5}$ & $1.46 \times 10^{-5}$ & $1.28 \times 10^{-5}$ & $7.05 \times 10^{-6}$ & $2.02 \times 10^{-5}$ \\
$10^{6}$ & $1.46 \times 10^{-7}$ & $1.28 \times 10^{-7}$ & $7.05 \times 10^{-8}$ & $2.02 \times 10^{-7}$ \\
$10^{7}$ & $1.46 \times 10^{-9}$ & $1.28 \times 10^{-9}$ & $7.05 \times 10^{-10}$ & $2.02 \times 10^{-9}$ \\
\hline
\end{tabular}

Table 1: QRPA estimates of the relevant combination of nuclear matrix elements, $\mathcal{M}_{\mathrm{GTF}}=$ $\mathcal{M}_{\mathrm{GT}}-\mathcal{M}_{\mathrm{F}}$, as a function of the KK neutrino mass $m_{(n)}$.

generally leads to predictions for $\langle m\rangle$ that depend on the double beta emitter isotope used in experiment. However, the difference in the predictions is too small for the higherdimensional singlet-neutrino models to be able to operate as a smoking gun for different $0 \nu \beta \beta$-decay experiments.

To obtain realistic predictions for the double beta decay observable $\langle m\rangle$, we have used the general formula $\left(\bar{\beta}_{\overline{3}} \overline{8}_{i}^{\prime}\right)$, where the infinite sum over $n$ has been truncated at $\left|n_{\max }\right|=$ $M_{F} R$, namely at the quantum gravity scale $M_{F}$.

In Table $\beth_{1}^{\bar{\eta}}$, we present numerical values for the difference of the nuclear matrix elements, $\mathcal{M}_{\mathrm{GTF}}=\mathcal{M}_{\mathrm{GT}}-\mathcal{M}_{\mathrm{F}}$, as a function of the KK neutrino mass $m_{(n)}$. Our estimates are obtained within the so-called Quasi-particle Random Phase Approximation

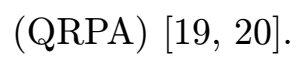

In Table ${ }_{2}^{2}$ - we show numerical values for the effective Majorana-neutrino mass $\langle m\rangle$ as derived for different nuclei in a 5-dimensional brane-shifted model, with $m=10 \mathrm{eV}$, 


\begin{tabular}{|c|c|c|c|c|c|c|c|c|}
\hline $1 / a$ & \multicolumn{8}{|c|}{$\langle m\rangle[\mathrm{eV}]$} \\
\hline$[\mathrm{GeV}]$ & ${ }^{76} \mathrm{Ge}$ & ${ }^{82} \mathrm{Se}$ & ${ }^{100} \mathrm{Mo}$ & ${ }^{116} \mathrm{Cd}$ & ${ }^{128} \mathrm{Te}$ & ${ }^{130} \mathrm{Te}$ & ${ }^{136} \mathrm{Xe}$ & ${ }^{150} \mathrm{Nd}$ \\
\hline 0.05 & 0.009 & 0.010 & 0.016 & 0.012 & 0.009 & 0.008 & -0.004 & -0.004 \\
\hline 0.1 & 0.052 & 0.054 & 0.061 & 0.062 & 0.052 & 0.050 & 0.025 & 0.026 \\
\hline 0.2 & 0.096 & 0.100 & 0.109 & 0.114 & 0.097 & 0.094 & 0.058 & 0.061 \\
\hline 0.3 & 0.123 & 0.128 & 0.136 & 0.143 & 0.124 & 0.121 & 0.082 & 0.086 \\
\hline 1 & 0.271 & 0.275 & 0.280 & 0.287 & 0.272 & 0.269 & 0.241 & 0.243 \\
\hline 10 & 0.493 & 0.493 & 0.494 & 0.495 & 0.493 & 0.493 & 0.489 & 0.489 \\
\hline $10^{2}$ & \multicolumn{8}{|c|}{0.513} \\
\hline $10^{3}$ & \multicolumn{8}{|c|}{0.535} \\
\hline $10^{4}$ & \multicolumn{8}{|c|}{0.066} \\
\hline $10^{10}$ & \multicolumn{8}{|c|}{$\lesssim 10^{-6}$} \\
\hline
\end{tabular}

Table 2: Numerical estimates of $\langle m\rangle$ for different nuclei in a 5-dimensional brane-shifted model, with $m=10 \mathrm{eV}, 1 / R=300 \mathrm{eV}, \varepsilon=1 /(4 R), \phi_{h}=-\pi / 4$ and $M_{F}=1 \mathrm{TeV}$.

$1 / R=300 \mathrm{eV}, \varepsilon=1 /(4 R), \phi_{h}=-\pi / 4$ and $M_{F}=1 \mathrm{TeV}$. In addition, we have varied discretely the brane-shifting scale $1 / a$ from $0.05 \mathrm{GeV}$ up to values much larger than $M_{F}$. It is obvious that, within the extra-dimensional framework considered, a sizeable value for $\langle m\rangle$ in the presently explorable range, is possible. Finally, for very small values of $a$, i.e. for $a \ll 1 / M_{F}$, we obtain the undetectably small result for the unshifted brane $a=0$.

Apart from explaining the recent excess in $0 \nu \beta \beta$ decays, the 5 -dimensional model with a small but non-vanishing shifted brane exhibits another very important property. The effective Majorana-neutrino mass $\langle m\rangle$ can be several orders of magnitude larger than the light neutrino mass $m_{\nu}$, for certain choices of the parameters $\varepsilon$ and $\phi_{h}$. To understand this phenomenon, let us first consider the eigenvalue equation $\left(\overline{2}-\overline{2} \overline{1}^{i}\right)$ for $\lambda=m_{\nu}$, written in the form:

$$
m_{\nu}+\sum_{n=-\infty}^{\infty} \frac{m^{(n) 2}}{\varepsilon+\frac{n}{R}-m_{\nu}}=0 .
$$

The infinite sum over KK neutrino states can be performed with the help of (2.22). Especially for $a=\pi R / q$ with $q$ being an integer much larger than 1, i.e. for $1 / M_{F} \ll a \lesssim$ $1 / q_{F}$, the light neutrino mass $m_{\nu}$ is given by

$$
m_{\nu} \approx-\pi m^{2} R\left[\cos ^{2} \phi_{h} \cot (\pi R \varepsilon)+\frac{1}{2} \sin \left(2 \phi_{h}\right)\right] .
$$

It is now easy to see that the light neutrino mass $m_{\nu}$ can be very suppressed for specific values of $\phi_{h}$ and $\varepsilon$. For instance, one obvious choice would be $\phi_{h} \approx-\pi / 4$ and $\varepsilon \approx 1 /(4 R)$. On the other hand, the effective neutrino mass $\langle m\rangle$ is determined by the second sinedependent term in (13.10in), which is induced by brane-shifting effects. Unlike the suppressed light neutrino mass $m_{\nu}$, the effective neutrino mass $\langle m\rangle$ can be sizeable in the observable range of several tenths of an $\mathrm{eV}$. This loss of correlation between the quantities $\langle m\rangle$ and $m_{\nu}$ is a rather unique feature of our higher-dimensional brane-shifted scenario. It provides 
an elegant mechanism for allowing the claimed evidence for a non-zero $0 \nu \beta \beta$ signal $[\underline{9}]$ to coexist with stringent cosmological constraints on the absolute neutrino mass scale from the

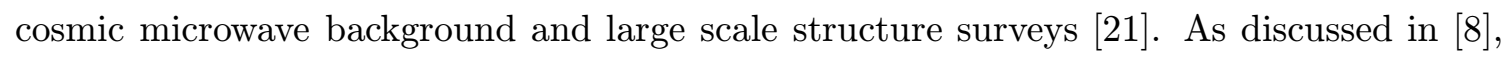
the above de-correlation property also plays a key rôle in model-building of 5-dimensional brane-shifted scenarios that could explain the neutrino oscillation data.

\section{Conclusions}

We have studied the model-building constraints derived from the requirement that KK singlet neutrinos in theories with large extra dimensions can give rise to a sizeable $0 \nu \beta \beta$ decay signal [8].]. Our analysis has been focused on 5-dimensional $S^{1} / Z_{2}$ orbifold models with one sterile (singlet) neutrino in the bulk, while the SM fields are considered to be localized on a 3-brane. In our model-building, we have also allowed the 3-brane to be displaced from the $S^{1} / Z_{2}$ orbifold fixed points. Within this minimal 5-dimensional brane-shifted framework, lepton-number violation can be introduced through Majorana-like bilinears, which may or may not arise from the Scherk-Schwarz mechanism, and through leptonnumber-violating Yukawa couplings. However, lepton-number-violating Yukawa couplings can be admitted in the theory, only if the 3-brane is shifted from the $S^{1} / Z_{2}$ orbifold fixed points. Apart from a possible stringy origin [i $\overline{1}_{L} \bar{z}_{1}$, brane-shifting might also be regarded as an effective result owing to a non-trivial 5-dimensional profile of the Higgs particle [20 $2 \overline{2}]$ and/or other SM fields [203i, $\left.2 \overline{2}_{2}^{4}\right]$ that live in different locations of a 3-brane with non-zero thickness which is centered at one of the $S^{1} / Z_{2}$ orbifold fixed points.

One major difficulty of the higher-dimensional theories is their generic prediction of a KK neutrino spectrum of approximately degenerate states with opposite CP parities that lead to exceedingly suppressed values for the effective Majorana-neutrino mass $\langle m\rangle$. Nevertheless, we have shown that within the 5-dimensional brane-shifted framework, the KK neutrinos can couple to the $W^{ \pm}$bosons with unequal strength, thus avoiding the disastrous CP-parity cancellations in the $0 \nu \beta \beta$-decay amplitude. In particular, the braneshifting parameter $a$ can be determined from the requirement that the effective Majorana mass $\langle m\rangle$ is in the observable range. In this way, we have found that $1 / a$ has to be larger than the typical Fermi nuclear momentum $q_{F}=100 \mathrm{MeV}$ and much smaller than the quantum gravity scale $M_{F}$, or equivalently $1 / M_{F} \ll a \lesssim 1 / q_{F}$.

An important prediction of our 5-dimensional brane-shifted model is that the effective Majorana-neutrino mass $\langle m\rangle$ and the scale of light neutrino masses can be completely decorrelated for certain natural choices of the Majorana-like bilinear term $\varepsilon$ and the original 5-dimensional Yukawa couplings $h_{1}^{l}$ and $h_{2}^{l}$ in (2, $\left.{ }^{2}-4\right)$. For example, if $\varepsilon \approx 1 /(4 R)$ and $h_{1}^{l} \approx-h_{2}^{l}$, we obtain light-neutrino masses that can be several orders of magnitude smaller than $\langle m\rangle$.

\section{References}

[1] N. Arkani-Hamed, S. Dimopoulos and G. Dvali, Phys. Lett. B429 (1998) 263; I. Antoniadis, N. Arkani-Hamed, S. Dimopoulos and G. Dvali, Phys. Lett. B436 (1998) 257; K.R. Dienes, E. Dudas and T. Gherghetta, Phys. Lett. B436 (1998) 55; Nucl. Phys. B537 (1999) 47; L. 
Randall and R. Sundrum, Phys. Rev. Lett. 83 (1999) 3370. For earlier studies, see I. Antoniadis, Phys. Lett. B246 (1990) 377; J.D. Lykken, Phys. Rev. D54 (1996) 3693; P. Hořava and E. Witten, Nucl. Phys. B460 (1996) 506; Nucl. Phys. B475 (1996) 94.

[2] K.R. Dienes, E. Dudas and T. Gherghetta, Nucl. Phys. B557 (1999) 25.

[3] N. Arkani-Hamed, S. Dimopoulos, G. Dvali and J. March-Russell, hep-ph/9811448.

[4] A. Pilaftsis, Phys. Rev. D60 (1999) 105023.

[5] A. Ioannisian and A. Pilaftsis, Phys. Rev. D62 (2000) 066001.

[6] M. Doi, T. Kotani and E. Takasugi, Prog. Theor. Phys. Suppl. 83 (1985) 1.

[7] For example, see the textbook by K. Grotz and H.V. Klapdor, "The Weak Interaction in Nuclear, Particle und Astrophysics," (Adam Hilger, Bristol, 1989).

[8] G. Bhattacharyya, H. V. Klapdor-Kleingrothaus, H. Päs and A. Pilaftsis, Phys. Rev. D67 (2003) 113001.

[9] H.V. Klapdor-Kleingrothaus, A. Dietz, H.L. Harney, I.V. Krivosheina, Mod. Phys. Lett. A16 (2001) 2409; H.V. Klapdor-Kleingrothaus, A. Dietz and I. Krivosheina, Particles and Nuclei 110 (2002) 57; Foundations of Physics 32 (2002) 1181; H.V. Klapdor-Kleingrothaus,

A. Dietz, I.V. Krivosheina, C. Doerr and C. Tomei, Phys. Lett. B578, 54 (2004) 54. .

[10] C. E. Aalseth et al. [IGEX Collaboration], Phys. Rev. D65 (2002) 092007.

[11] F. Piquemal [NEMO Collaboration], hep-ex/0205006.

[12] S. R. Elliott and P. Vogel, Ann. Rev. Nucl. Part. Sci. 52, 115 (2002).

[13] R.N. Mohapatra, A. Perez-Lorenzana and C.A. de S. Pires, Phys. Lett. B491 (2000) 143.

[14] S.J. Huber and Q. Shafi, Phys. Lett. B544 (2002) 295.

[15] J. Scherk and J.H. Schwarz, Phys. Lett. B82 (1979) 60; Nucl. Phys. B153 (1979) 61;

P. Fayet, Phys. Lett. B159 (1985) 121; Nucl. Phys. B263 (1986) 649.

[16] A. Delgado, G. v. Gersdorff and M. Quiros, hep-th/0210181; see, also, J.A. Bagger, F. Feruglio and F. Zwirner, Phys. Rev. Lett. 88 (2002) 101601.

[17] For example, see, E.G. Gimon and J. Polchinski, Phys. Rev. D54 (1996) 1667.

[18] M. Hirsch and H.V. Klapdor-Kleingrothaus, in Proc. "Double Beta Decay and Related Topics," Trento 1995, World Scientific, p. 175.

[19] K. Muto, E. Bender and H.V. Klapdor, Z. Phys. A334 (1989) 187.

[20] A. Staudt, K. Muto and H.V. Klapdor-Kleingrothaus, Europhys. Lett. 13 (1990) 31;

M. Hirsch, K. Muto, T. Oda and H.V. Klapdor-Kleingrothaus, Z. Phys. A347 (1994) 151.

[21] G. Bhattacharyya, H. Päs, L. Song and T.J. Weiler, Phys. Lett. B B564 (2003) 175.

[22] V.A. Rubakov and M.E. Shaposhnikov, Phys. Lett. B125 (1983) 136.

[23] N. Arkani-Hamed and M. Schmaltz, Phys. Rev. D61 (2000) 033005; E.A. Mirabelli and M. Schmaltz, Phys. Rev. D61 (2000) 113011; G. Dvali and M. Shifman, Phys. Lett. B475 (2000) 295; G.C. Branco, A. de Gouvea and M.N. Rebelo, Phys. Lett. B506 (2001) 115; M. Raidal and A. Strumia, hep-ph/0210021.

[24] H.V. Klapdor-Kleingrothaus and U. Sarkar, Phys. Lett. B541 (2002) 332. 